\title{
Correction to: MEN2-related pheochromocytoma: current state of knowledge, specific characteristics in MEN2B, and perspectives
}

\author{
Vincent Amodru ${ }^{1} \cdot$ David Taieb $^{2} \cdot$ Carole Guerin $^{3} \cdot$ Pauline Romanet $^{4} \cdot$ Nunzia Paladino $^{3} \cdot$ Thierry Brue $^{1}$. \\ Thomas Cuny $^{1} \cdot$ Anne Barlier $^{4} \cdot$ Frederic Sebag $^{3} \cdot{\text { Frederic Castinetti }{ }^{1}{ }^{1}}^{1}$
}

Published online: 2 June 2020

(c) Springer Science+Business Media, LLC, part of Springer Nature 2020

Correction to: Endocrine

https://doi.org/10.1007/s12020-020-02332-2

The original version of this article unfortunately contained a mistake. The given and family names of all the authors are transposed.
The author group has been updated above and the original article has been corrected.

The original article can be found online at https://doi.org/10.1007/ s12020-020-02332-2.

Frederic Castinetti

Frederic.castinetti@ap-hm.fr

1 Department of Endocrinology, Aix-Marseille University, INSERM U1251, Endo-ERN Reference Center for Rare Genetic Tumor Syndromes, Assistance Publique-Hopitaux de Marseille, Marseille, France

2 Department of Nuclear Medicine, Aix-Marseille University, EndoERN Reference Center for Rare Genetic Tumor Syndromes, Assistance Publique-Hopitaux de Marseille, Marseille, France

3 Department of Endocrine Surgery, Aix-Marseille University, Endo-ERN Reference Center for Rare Genetic Tumor Syndromes, Assistance Publique-Hopitaux de Marseille, Marseille, France

4 Department of Molecular Biology, Aix-Marseille University, INSERM U1251, Endo-ERN Reference Center for Rare Genetic Tumor Syndromes, Assistance Publique-Hopitaux de Marseille, Marseille, France 